\title{
NEW EVIDENCE OF HETEROSIS IN NATURALLY OCCUR- RING INVERSION HETEROZYGOTES IN DROSOPHILA PSEUDOOBSCURA
}

\author{
HOWARD LEVENE * and THEODOSIUS DOBZHANSKY $\dagger$ \\ Departments of Mathematical Statistics and Zoology, Columbia University
}

Received 26.iii.57

\section{REVIEW OF THE PROBLEM}

THE occurrence in natural populations of Drosophila of inversion heterozygotes has long been known. Positive evidence that this chromosomal polymorphism has an adaptive function came with the finding that the frequencies of certain gene arrangements in some populations of Drosophila pseudoobscura undergo cyclic changes which follow the annual succession of seasons (Dobzhansky, 1943). Wright and Dobzhansky (1946) then made experimental populations in which progenies of flies collected in nature were allowed to breed for a series of generations in population cages. The proportions of chromosomes with different gene arrangements were observed to change from generation to generation. In populations of uniform geographic origin (i.e. composed of progenies of flies collected in the same locality) the changes usually obeyed a simple rule. Apparently stable equilibria became established, at which the chromosomes with different gene arrangements continued to occur in the populations. This behaviour is consistent with the hypothesis that the chromosomal polymorphism is balanced; the inversion heterozygotes are heterotic, and the corresponding homozygotes are adaptively inferior to the heterozygotes. Later it was found (Dobzhansky, 1950) that in geographically mixed populations (derived from flies collected in different localities) heterosis is no longer the rule; the heterozygotes may be equal, superior, or inferior to the homozygotes.

The hypothesis of heterosis was verified both in experimental and in natural populations. Dobzhansky (1947) compared the frequencies of inversion homo- and heterozygotes among individuals grown under optimal conditions with those among flies which developed in severely crowded population cages. The frequencies obeyed the binomial square rule among the former, but among the latter there was an excess of heterozygotes and a deficiency of homozygotes. Dobzhansky and Levene (1948) then compared the frequencies of inversion homoand heterozygotes among eggs deposited by wild-caught females of Drosophila pseudoobscura, and among adult males of the same species

* Research under Contract No. Nonr- 266(33), Project No. 042-034, United States Office of Naval Research.

$\dagger$ Research carried out under Contract No. AT-(30-I)-1 I5I, United States Atomic Energy Commission. 
collected in their natural habitats. The binomial square rule was obeyed quite well in the egg samples. Among the wild-caught males, the deviations from the proportions demanded by the binomial square rule were not statistically significant in most samples. But the combined data, for males collected in 22 localities mostly in California, showed a quite significant excess of heterozygotes. This is as expected if the inversion heterozygotes are favoured by differential survival in most populations most of the time.

Da Cunha (1953) found some natural populations of Drosophila willistoni in which more than $5^{\circ}$ per cent. of the females are heterozygous for certain inversions in the $\mathrm{X}$-chromosomes. To be sure, frequencies of heterozygotes in excess of $5^{\circ}$ per cent. may be observed without heterosis in populations which are in the throes of rapid genetic changes, and which therefore are not at equilibrium. This difficulty was removed when da Cunha (1956) found several inversions which maintained frequencies of heterozygotes in excess of $5^{\circ}$ per cent. in experimental populations of $D$. willistoni, even after these populations reached stable genetic equilibria. Dobzhansky and Pavlovsky (1955) described a population of $D$. tropicalis from a locality in Central America in which about 70 per cent. of the individuals were heterozygous for a certain inversion. An experimental laboratory population derived from the flies from this locality contained 96 per cent. of heterozygotes for the same inversion. Pavan, Dobzhansky, and da Gunha (1957) observed in a locality in Peru a population of $D$. paulistcrum with 79 per cent. of heterozygotes for a certain inversion. A somewhat lower frequency, about 60 per cent., of heterozygotes for another inversion was encountered in $D$. willistoni in north-eastern Brazil. The interest of this case is, however, heightened by the fact that the excess of heterozygotes was met with not in one but in several localities, the most remote ones separated by a distance of at least 200 kilometres, and in one of these localities the excess was ascertained at different seasons of the year.

It would certainly be wrong to conclude, as some authors did from the above data, that excesses of heterozygotes must be present at all times in all populations. The occurrence in some natural populations of rapid seasonal changes in the frequencies of chromosomes with different gene arrangements is in itself evidence that the relative fitness of at least some inversion homo- and heterozygotes varies in different environments. An extraordinary sensitivity to environmental changes was already found in Drosophila pseudoobscura by Wright and Dobzhansky (1946). At $25^{\circ} \mathrm{C}$., the fitness of a certain inversion heterozygote was more than double that of a certain homozygote, while at $16^{\circ} \mathrm{C}$. both were apparently alike in fitness. Conversely, certain karyotypcs in $D$. persimilis were about equal in fitness at $25^{\circ}$ but very different at $16^{\circ}$. In this connection, it is probably not accidental that $D$. pseudoobscura is more at home in warmer and $D$. persimilis in cooler habitats. Da Cunha (195I) and Dobzhansky and 
Spassky (1954) induced changes in the relative adaptive values of heterozygotes and homozygotes for some inversions in D. pseudoobscura by feeding experimental populations on different species of yeasts. When fed on a nutrient medium with a certain species of yeast, a normally heterotic inversion heterozygote became adaptively inferior to one of the homozygotes at $21^{\circ}$, although not at $25^{\circ}$ nor at $16^{\circ}$. As a consequence, stable genetic equilibria were cstablished in some but not in other experimental populations. Yet, no differences in the behaviour of experimental populations of $D$. willistoni fed on different species of yeasts were detected by da Cunha (1956).

Perhaps the most striking demonstration of a delicate sensitivity of inversion homo- and heterozygotes in Drosophila pseudoobscura to the environment was adduced in experimental populations in which as many as six different karyotypes were simultaneously present (Levene, Pavlovsky, Dobzhansky, 1954). The adaptive value of a karyotype depends not only on such variables as temperature and food but also on what other karyotypes are living in the same medium. Thus, A may be superior to B in the absence of $\mathrm{C}$, but it may become inferior to $\mathrm{B}$ when $\mathrm{C}$ is fresent. A different kind of sensitivity was discovered in $D$. robusta (Levitan, 1951, 1954, 1955). The gene arrangements in the two limbs of a metacentric chromosome show, in at least some populations of this species, a non-random association, which suggests that the chromosome acts as an organised unit rather than as a mechanical aggregate of genes. Moreover, this nonrandomness is more pronounced in males than in females; seasonal changes in the frequencies of the gene arrangements are also more evident in the male than in the female sex. It should also be noted that in $D$. pseudoobscura and in $D$. persimilis seasonal changes occur in populations of some geographic localities but not in others (Dobzhansky, I 956 and older work). It certainly does not follow that the carriers of the different karyotypes are differentiated in fitness in the former but not in the latter localities.

The purpose of the present article is to report some new, and quite uncomplicated, evidence that the heterozygotes for chromosomal inversions naturally occurring in Drosophila pseudoobscura and Drosophila persimilis are indeed superior in fitness to the corresponding homozygotes.

\section{THE DATA}

Strains of Drosophila pseudoobscura and $D$. persimilis have been maintained for a number of years in our laboratory. With rare exceptions, each strain is derived from a single fertilised female collected in the natural habitats of the respective species, mostly by one of the authors (Th. D.). The dates of collecting have been recorded. The chromosomal constitution which these strains possessed soon after their establishment in the laboratory is also recorded on the basis of the examination of the giant chromosomes in the larval salivary 
glands. Some of the strains carried one or more kinds of heterozygotes for inversions in the third chromosomes ; this is, of course, as expected, since at the start each strain must have contained at least four third chromosomes, two derived from the original female, and two from the original male progenitor. The question, then, is this : Does the chromosomal polymorphism persist in the laboratory strains for as long or longer than it may be expected to persist owing to chance alone?

Laboratory strains are maintained by periodic transfer of samples of flies from the old culture bottles to fresh cultures; therefore, the unfixed genetic variants in each strain are exposed in every generation to the risk of not being included among the parents of the succeeding generation. If these variants are adaptively neutral, the risk is a function of the number of flies of the preceding generation which become the parents of the succeeding generation. This number can be only approximately estimated, because for maintenance of routine laboratory stocks the flies are transferred without being counted. Mr B. Spassky, who has maintained most of these stocks during most of the time which they have been kept in this laboratory, estimates that the average numbers of the flies transferred from culture to culture were close to twenty, and the maximum numbers close to forty, in each case about equally divided between females and males. For our present purposes an overestimate of this number is preferable to an underestimate; we shall, therefore, use both the probable and the upper estimates in the calculations. The strains are normally kept in a constant temperature room at about $16^{\circ} \mathrm{C}$., but most of them werc kept for shorter or longer periods at room temperatures, especially during the early years. They are transferred to fresh cultures about once in six weeks. We shall use six weeks as the estimate of the length of a generation, although when the strains were kept at room temperatures they had to be transferred at shorter intervals.

In November I 956 samples of eggs deposited by the flies from the different strains were taken, and placed in culture bottles in a way to avoid overpopulation and crowding of the larvæ. Salivary glands from about ten larvæ from each strain, one gland per larva, were stained in acetic orcein. The preparations were made by Mrs N. Spassky; the gene arrangements in the third chromosomes were examined and recorded by one of us (Th. D.). The results are summarised in table $I$.

Table I lists 28 strains of Drosophila pseudoobscura and I I strains of $D$. persimilis. For each strain are given the name of the locality in which the wild progenitor was collected, the date of collecting, an estimate of the number of generations during which the strain was kept in the laboratory, and the gene arrangements in the third chromosomes which were found in November of 1956 . For descriptions of these gene arrangements, and for drawings of the chromosome configurations in some of the inversion heterozygotes, see Dobzhansky 
(I944). In only three strains, marked in table I by asterisks, have the results of the examination in 1956 deviated from those of the

TABLE I

The gene arrangements in the third chromosomes in laboratory strains of Drosophila pseudoobscura and Drosophila persimilis

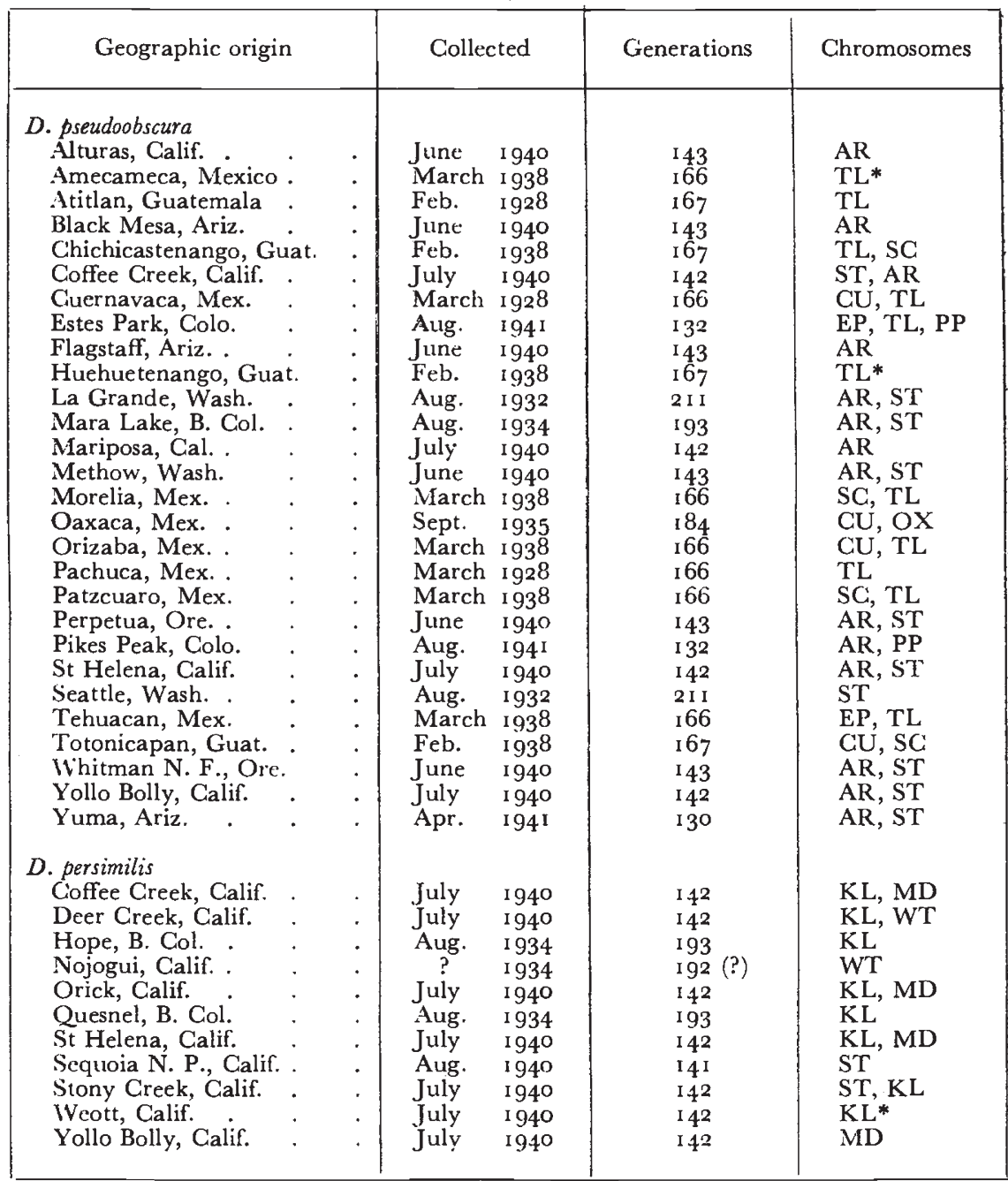

AR-Arrowhead, CU-Cuernavaca, EP_Estes Park, KL-Klamath, MD_-Mendocino, OX-Oaxaca, PP-Pikes Peak, SC-Santa Cruz, ST-Standard, TL-Tree Line, WTWhiteney.

original examination. In all three cases, two different arrangements were originally found but only one was still extant in 1956. More precisely, the Amecameca, Mexico, strain contained in $193^{8} \mathrm{CU}$ as well as TL chromosomes, but only TL has been preserved. In the Huehuetenango, Guatemala, strain TL and SC chromosomes were present in I938, but SC was lost. In the $W^{+}$cott, California, strain 
of Drosophila persimilis $\mathrm{KL}$ and $\mathrm{MD}$ chromosomes were found in $\mathrm{I} 94^{\circ}$, and only $\mathrm{KL}$ in 1956 . In 12 strains, only a single third chromosome gene arrangement was found at the original examination, and the same chromosome structure was seen again in 1956. In 23 strains, two different third chromosomes were originally. found, and the same two found again in 1956 ; and in $\mathrm{I}$ strain three different third chromosomes were found and recovered again. Most of the strains have, evidently, maintained their chromosomal composition during more than roo generations which they spent in the laboratory cultures.

\section{MATHEMATICAL ANALYSIS}

Sewall Wright (193I) investigated inbreeding in a random r.ating population. If the population consists of $\mathrm{N}_{m}$ males and $\mathrm{N}_{f}$ females the effective population size, $\mathrm{N}$, is defined by $\mathrm{I} / \mathrm{N}=\mathrm{I} /\left({ }_{4} \mathrm{~N}_{m}\right)+\mathrm{I} /\left({ }_{4} \mathrm{~N}_{f}\right)$. If $\mathrm{N}_{m}=\mathrm{N}_{f}, \mathrm{~N}=\mathrm{N}_{m}+\mathrm{N}_{f}$. In the present material, each culture was started with $\mathrm{N}^{\prime}$ flies chosen at random from the previous culture. Thus $\mathrm{N}_{m}$ fluctuated at random about a mean value of $\mathrm{N}^{\prime} / 2$, and because the sexes were not precisely equally frequent, the effective population size over a period of time is close to $N^{\prime}-r$. Since $N^{\prime}$ is only known very roughly, we can take $\mathrm{N}=\mathrm{N}^{\prime}$ with no serious error.

Wright (193I) gave a recurrence relation for the coefficient of inbreeding $F$, and for the panmictic index $P=\mathrm{I}-\mathrm{F}$. The relation for $\mathrm{P}$ is

$$
\mathrm{P}_{t}=\left(\mathrm{I}-\frac{\mathrm{I}}{\mathrm{N}}\right) \mathrm{P}_{t-1}+\frac{\mathrm{I}}{2 \mathrm{~N}} \mathrm{P}_{t-2}, \quad .
$$

where the subscript refers to the time in generations since the establishment of the population. The explicit solution of $(\mathrm{I})$ in terms of $\mathrm{N}$ and $t$ is easily obtained, and was first given, to the best of our knowledge, by Malécot (1946). The exact formula is

$$
\begin{aligned}
P_{t}= & \left(\frac{\mathrm{I}-\frac{\mathrm{I}}{\mathrm{N}}-\sqrt{\mathrm{I}-\frac{\mathrm{I}}{\mathrm{N}^{2}}}}{\sqrt[2]{\mathrm{I}-\frac{\mathrm{I}}{\mathrm{N}^{2}}}}\right)\left(\frac{\mathrm{I}-\frac{\mathrm{I}}{\mathrm{N}}+\sqrt{\mathrm{I}-\frac{\mathrm{I}}{\mathrm{N}^{2}}}}{2}\right)^{t}- \\
& \left(\frac{\mathrm{I}-\frac{\mathrm{I}}{\mathrm{N}}+3 \sqrt{\mathrm{I}-\frac{\mathrm{I}}{\mathrm{N}^{2}}}}{\sqrt[2]{\mathrm{I}-\frac{\mathrm{I}}{\mathrm{N}^{2}}}}\right)\left(\frac{\mathrm{I}-\frac{\mathrm{I}}{\mathrm{N}}-\sqrt{\mathrm{I}-\frac{\mathrm{I}}{\mathrm{N}^{2}}}}{2}\right)^{t}
\end{aligned}
$$

The exact value of $P_{0}$ and $P_{1}$ is $I$. For $t$ greater than $\mathrm{r}$, the simplified approximate formula

$$
\mathrm{P}_{t}=\left(\mathrm{I}+\frac{\mathrm{I}}{2 \mathrm{~N}}\right)\left(\mathrm{I}-\frac{\mathrm{I}}{2 \mathrm{~N}}\right)^{t}
$$

differs from the exact value by a quantity of the order of $t /\left(\mathrm{r}_{6} \mathrm{~N}^{4}\right)$, 
which can almost always be neglected. (E.g. for $\mathrm{N}$ as small as 20 and $t$ as large as 200 , the error is of the order $1 / 12,800 \cong .000$ I). The even simpler form

$$
\mathrm{P}_{t}=e^{-\frac{t}{2 N}}
$$

has an absolute error of at most $1 / 2 \mathrm{~N}$ and a relative error of approximately $(\mathrm{I} / 2 \mathrm{~N})-(t+\mathrm{I}) / 8 \mathrm{~N}^{2}$. Table 2 gives the estimated values of $t$, the number of elapsed generations, the number of cultures with cach such value, and the value of $P_{t}$ calculated from (3) for the most probable $\mathrm{N}, 20$, and for the upper bound of 40 .

It may be worth while to make a few remarks here about the nature of this "inbreeding" in random mating finite populations, since many non-mathematical geneticists find it a confusing subject. Let us suppose a population segregating for a pair of alleles or karyotypes $\mathrm{A}$ and $\mathrm{A}^{\prime}$, and suppose that in the founding population (generation $\mathrm{O}) \mathrm{A}$ has the frequency $q_{0}$ in both males and females. Then in the next generation $(t=\mathrm{I})$, the probability that an individual chosen at random is hetcrozygous is $2 q_{0}\left(1-q_{0}\right)$. The probability that an individual chosen at random from the $t$-th generation will be a heterozygote will be $\mathrm{P}_{t} \cdot 2 q_{0}\left(\mathrm{I}-q_{0}\right)$, and in this sense $\mathrm{P}_{t}$ measures the loss of heterozygosis due to inbreeding. However, in fact, the loss of heterozygosis is entirely due to genetic drift from random sampling errors, and if $q_{t}$ is the actual frequency of $\mathrm{A}$ in the $t$-th generation, then the probability that an individual chosen at random in the $t$-th generation will be a heterozygote is $2 q_{t-1}\left(\mathrm{I}-q_{t-1}\right)$. In other words the Hardy-Weinberg equilibrium will be maintained in the population, so long as it is calculated from current gene frequencies, and any " inbreeding" is only relative to a particular reference point in the past. This is quite different from the real departure from the HardyWeinberg equilibrium in a population where close relatives mate more frequently than would be expected by chance.

Thus the probability that a random individual will be homozygous is $\mathrm{I}-2 \mathrm{P}_{1} q_{0}\left(\mathrm{I}-q_{0}\right)$. But what is the probability that a second individual chosen at random will be, say, $\mathrm{A} \mathrm{A}$, given that the first one was $\mathrm{A} \mathrm{A}$; what is the probability that two individuals chosen at random will be homozygotes of the same kind? The answer to these questions is not obvious. It is even less obvious what the probability is that all the individuals in the $t$-th generation are homozygotes of the same kind; that is that $q_{t}=\mathrm{o}$ or I, although this probability must be large if $\mathrm{P}_{t}$ is close to zero. This probability can be obtained by the methods of Markoff chains, although the labour increases with $\mathrm{N}$, or for large $\mathrm{N}$ by consideration of a diffusion process. Kimura (r954) has given the solution for large $\mathrm{N}$ in the form

$$
\mathrm{H}_{t}=6 q_{0}\left(\mathrm{I}-q_{0}\right) e^{-t / 2 \mathrm{~N}}+\mathrm{I} 4 q_{0}\left(\mathrm{I}-q_{0}\right)\left[\mathrm{I}-5 q_{0}\left(\mathrm{I}-q_{0}\right)\right] e^{-6 t / 2 \mathrm{~N}}+\mathrm{R},
$$

where $\mathrm{H}_{t}$ is the probability that both alleles are still present and $\mathrm{R}$ is of the order of magnitude of $e^{-15^{t / 2} \mathrm{~N}}$. For $6 t / 2 \mathrm{~N}$ greater than 7 
or $t$ greater than $2 \cdot{ }_{4} \mathrm{~N}$, even the third term is less than $0 \cdot 00 \mathrm{I}$, and we have

$$
\mathrm{H}_{t}=6 q_{0}\left(\mathrm{I}-q_{0}\right) e^{-t / 2 \mathrm{~N}} .
$$

This probability of no fixation is largest if $q_{0}=\mathrm{I} / 2$, as would be expected on intuitive grounds, so that even in this least favourable case for fixation, the probability that fixation has not occurred is only

$$
\mathrm{H}_{t}=\frac{3}{2} e^{-t / 2 \mathrm{~N}}
$$

or approximately $\mathrm{I}_{5} \mathrm{P}_{t}$.

\section{TABLE 2}

Estimated number of generations, $t$, number of originally heterozygous cultures for each $t$, number of cultures no longer heterozygous, and values of the panmictic index $P_{\mathrm{t}}=I-F_{\mathrm{t}}$ and of the maximum probability of remaining heterozygous for the most probable popula-

\begin{tabular}{|c|c|c|c|c|c|c|}
\hline \multirow{2}{*}{$t$} & \multirow{2}{*}{$\begin{array}{l}\text { No. of } \\
\text { cultures }\end{array}$} & \multirow{2}{*}{$\begin{array}{c}\text { No. not } \\
\text { heterozygous }\end{array}$} & \multicolumn{2}{|c|}{$N=20$} & \multicolumn{2}{|c|}{$\mathrm{N}=4^{\circ}$} \\
\hline & & & $\mathbf{P}_{t}$ & Prob. het. & $P_{t}$ & Prob. het. \\
\hline 130 & I & 0 & 0.041 & 0.059 & 0.202 & $0 \cdot 296$ \\
\hline 132 & 2 & o & 0.040 & 0.056 & 0.197 & 0.288 \\
\hline $\begin{array}{l}142 \\
\end{array}$ & 9 & I & $0.03 \mathrm{I}$ & 0.044 & $0 \cdot 174$ & 0.255 \\
\hline 143 & 3 & o & 0.030 & 0.042 & 0.172 & 0.250 \\
\hline 166 & 6 & I & 0.017 & 0.024 & 0.129 & $0 \cdot 189$ \\
\hline $\begin{array}{l}\text { I } 67 \\
\text {. }\end{array}$ & 3 & I & 0.017 & 0.022 & 0.127 & o 186 \\
\hline I 84 & $\mathrm{I}$ & 0 & 0.011 & 0.015 & 0.103 & $0.15^{\circ}$ \\
\hline 193 & I & 0 & 0.009 & 0.012 & 0.092 & $0 \cdot 134$ \\
\hline $2 I I$ & I & o & 0.006 & 0.007 & 0.074 & $0 \cdot 106$ \\
\hline Total & 27 & 3 & & & & \\
\hline
\end{tabular}
tion size, $\mathcal{N}=20$, and the upper limit of population size, $\mathcal{N}=40$

Table 2 gives the value of $\mathrm{H}_{t}$, calculated from (7), for all cultures for $\mathrm{N}=20$ and $\mathrm{N}=40$. In table 2 the largest probability that both alleles are still present is for $\mathrm{N}=40, t=130$ and is approximately 0.3 . Even if we took this largest value as the actual probability for all the cultures, the probability that 24 out of the original 27 cultures with more than one chromosome present would not lose any chromosome is less than $10^{-7}$. If we take account of the actuat time values, and the fact that in one case all of three original chromoscmes were preserved, this probability would become negligible. Evidently then, some agency was acting to maintain heterozygosis, and the most likely candidate is selection in favour of heterozygotes.

\section{INBREEDING WHEN HETEROZYGOTES ARE FAVOURED}

It is clear that when heterozygotes are favoured by selection, progress toward homozygosis under inbreeding will be greatly slowed. This process has been studied by the methods of Markoff chains by 
Hayman (1953), Hayman and Mather (1953 and 1956), Reeve (1955), and Haldane (1956). Most of these authors considered regular systems of inbreeding, and the only connection with the present problem is that sib mating is equivalent to $\mathrm{N}_{m}=\mathrm{N}_{f}=\mathrm{I}$. Kimura (1955) has considered random mating in a finite population with genic selection, but not selection in favour of heterozygotes. The Markoff chain methods become excessively laborious for $\mathrm{N}$ as large as 20 , involving $40 \times 40$ matrices, while Kimura's methods assume large $\mathrm{N}$ and might not be valid for $\mathrm{N}=20$, even if the formidable analytic difficulties were overcome. Accordingly recourse was had to Monte Carlo methods or in less elegant terms " bean bag genetics", that is, to a.sampling experiment. The model chosen gives the karyotypes $\mathrm{A} \mathrm{A}, \mathrm{A} \mathrm{A}^{\prime}, \mathrm{A}^{\prime} \mathrm{A}^{\prime}$, in the ratio $(\mathrm{I}-s): \mathrm{I}:\left(\mathrm{I}-s^{\prime}\right)$. Then if the gene frequency in the $t$-th generation is $q_{t}$, the expected gene frequency in the $(t+\mathrm{I})-$ th generation is

$$
\tilde{q}_{t+1}=\frac{q_{t}-s q_{t}^{2}}{\mathrm{I}-s q^{2}-s^{\prime}(\mathrm{I}-q)^{2}}
$$

For a given value of $s$, the probability of fixation in a large number of generations is least for $s^{\prime}=s$, since in this case equilibrium is at $q=\mathrm{I} / 2$ and large or small values of $q$, with danger of fixation, are least likely. Accordingly $s^{\prime}$ was chosen to equal $s$, and (8) became

$$
\tilde{q}_{t+1}=\frac{q_{t}-s q_{t}^{2}}{\mathrm{I}-s\left[q^{2}+(\mathrm{I}-q)^{2}\right]}
$$

To minimise the chance of fixation, $\tilde{q}_{0}$ was then chosen to be $1 / 2$, and a sample of $2 \mathrm{~N}$ was chosen from a binomial population with probability $1 / 2$. The resulting frequency of A was $q_{1}$. Then $\tilde{q}_{2}$ was calculated from (9) and a sample of $2 \mathrm{~N}$ was chosen from a binomial population with the proportion $\tilde{q}_{2}$ of A's. The result of this sampling was the observed value of $q_{2}$, from which $\tilde{q}_{3}$ was calculated, and so on. The process was stopped after i 70 generations (as representative of the actual cultures) unless fixation was reached before that time.

For $\mathrm{N}=4^{\circ}$, and $s=s^{\prime}=0 \cdot 1$, five trial populations reached fixation in 22, 29, 37, 45 and 55 "generations". This result is significantly different from the observed 3 out of 27 using Fisher's exact test $(\mathrm{P}=0.0002)$. Evidently an advantage of ro per cent. for the heterozygote is not enough to prevent fixation, even with the upper limit of 40 for the population size and with $q_{0}=1 / 2$, the value least favourable to fixation. Next the more probable population sizc of 20 was chosen, and $s=s^{\prime}$ increased to $1 / 2$. Now two trials went 170 generations without reaching fixation. Finally two trials with $\mathrm{N}=20$ and $s=s^{\prime}=1 / 4$ gave no fixation in 170 generations, although a third reached fixation in only if generations. In general if $q$ became small at some time $t$, but did not reach fixation, selection soon returned $q$ to near $\mathrm{I} / 2$, so that the four artificial populations 
that did not reach equilibrium in 170 generations ended with the rarer allele having a $q$ of $0 \cdot 35,0 \cdot 40,0 \cdot 42$, and $0 \cdot 45$. Further trials with $s=I / 2$ and $s=I / 4$ were not made because of the labour and the fact that very many more trials would have had to be made before a significant difference from the observed proportion of 3 fixed out of 27 could be found. Thus it was not practicable to distinguish between $s=I / 2$ and $s=I / 4$ as the proper value, although $s=0 \cdot 1$ was definitely too small. All that can safely be said is that for most of the chromosome pairs studied $s$ and $s^{\prime}$ must have been greater than $0 \cdot I$ and probably greater than $0 \cdot 2$. The three cultures that reached fixation may have been "unlucky" or may have had smaller values of $s$ and $s^{\prime}$, or $s$ and $s^{\prime}$ of opposite sign.

\section{DISCUSSION}

In Drosophila pseudoobscura and in D. persimilis, heterozygotes which carry two chromosomes of a pair with different gene arrangements but derived from the same geographic population are usually heterotic. However, Epling, Mitchell and Mattoni (1953, 1957) put forward what they believe to be an alternative hypothesis. It is well known that reduction of crossing over in a certain chromosome by a heterozygous inversion may be accompanied by increased recombination in some portions of the chromosomes in which crossing over is normally rare. Epling, Mitchell and Mattoni then postulate that under changing environmental conditions, the increased recombination in the residual genome of structural heterozygotes may aid in adapting to the changing conditions and consequently the offspring of structural heterozygotes will be favoured. It seems doubtful whether a second order effect such as this could actually give the heterozygotes as much advantage as they have in natural populations. As an explanation of the retention of the inversion heterozygotes under the fairly constant conditions of the laboratory cultures, the hypothesis of Epling et al. may safely be dismissed.

Nevertheless, heterosis is not an intrinsic propcrty of inversion heterozygotes. Some inversions failed to display heterosis in experimental populations of Drosophila pseudoobscura kept at $16^{\circ} \mathrm{C}$., or fed on nutrient media with certain yeasts (Dobzhansky and Spassky, 1954 and other work, see above). Yet it is exactly at that temperature that our laboratory strains have been kept and showed a persistence of the inversion polymorphism. This is not necessarily a contradiction ; relatively weak heterosis is hard to detect in experimental populations, and different geographic strains may have different temperature sensitivity ranges, just as the related species, $D$. pseudoobscura and $D$. persimilis, are known to have. It is even less necessary to suppose, as some writers have done, that the heterosis will lead to differential mortality of the inversion homozygotes and consequent disturbances of the ratios demanded by the binomial square rule. Such disturbances 
have indeed been found in certain populations, both natural and experimental ones, but they are neither expected nor observed to be universal. In fact, an advantage of the heterozygote with respect to such factors as fertility and fecundity would not cause such disturbances.

Only a few studies have separated the individual components of adaptive value. Spiess, Ketchel and Kinne (1952) found the WT/KL inversion heterozygotes in Drosophila persimilis appreciably superior to the WT/WT and $\mathrm{KL} / \mathrm{KL}$. homozygotes in fecundity. Spiess and Schuellein (1956) showed further that the WT/KL heterozygotes exceed the homozygotes in speed of development from egg to adult and in survival rates during the development. Finally, the heteroz.ygotes possessed superior homeostatic buffering, expressed in lower environmental components of the variance estimates for the traits studied. Such superior homeostasis has also been found by RosenbaumMoos (1955) in D. pseudoobscura inversion heterozygotes. In this species, the over-all fitness of the ST/CH heterozygotes was found to be no higher than that of the ST/ST homozygotes, but much higher than that of the $\mathrm{CH} / \mathrm{CH}$ homozygotes. However, the different components of fitness of these karyotypes are known to vary in their relative values quite appreciably in different environments (Heuts, 1947, I948; Birch, I955).

Inversion polymorphism is clearly a part of the adaptive machinery in natural populations of Drosophila. But no organic system is adaptive as such; adaptedness is harmony between a living system and the conditions of its existence. The relative adaptive values of the heterozygous and homozygous karyotypes in Drosophila populations are exquisitely sensitive to variations in the environment; and yet the populations are homeostatically buffered against environmental disturbances because they are composed of numerous genetic types with diverse environmental optima.

\section{SUMMARY}

Strains of Drosophila pseudoobscura and Drosophila persimilis, each descended from a single inseminated female collected in nature, have been kept in laboratory cultures for an estimated I 30 to 2 I I generations (see table I for particulars). The strains were examined for inverted sections in their third chromosomes within a few generations after their capture. At that time, 26 strains contained two gene arrangements each, 12 strains one gene arrangement each, and I strain three gene arrangements. Re-examination of the same strains showed that only 3 of the 27 strains which were structurally polymorphic became uniform in the course of time.

Groups of flies estimated to consist of between 20 and 40 individuals were transferred to new food in each generation to maintain the strains. It can be calculated that, with this breeding system, the strains should have become homozygous with respect to most of the unfixed genes 
and chromosomal structures which they contained originally. The failure of the 24 out of the 27 strains to become homozygous for the gene arrangements in third chromosomes shows that the inversion heterozygotes are superior in fitness to the inversion homozygotes.

Acknowledgments.-The strains which served as material for the present investigation have been maintained for more than twenty years chiefly by Mr Boris Spassky and by Mrs N. Spassky. Mrs Spassky also prepared the slides for the examination of the chromosomes in the salivary gland cells. We are obligated also to Prof. Carl Epling for permission to read the manuscript of his paper before its publication.

\section{REFERENCES}

BIRCH, L. C. I955. Selection in Drosophila pseudoobscura in relation to crowding. Evolution, 9, 389-399.

DA CUNHA, A. B. I95 I. Modification of the adaptive values of chromosomal types of Drosophila pseudoobscura by nutritional variables. Evolution, 5, 395-404.

DA GUNHA, A. B. 1953. Chromosomal inversions with sex-limited effects. Nature, 172,8 I 5 .

DA CUNHA, A. в. 1956. Differential viability favoring inversion heterozygotes in Drosophila willistoni. Evolution, Io, 23 1-234.

Dobzhansky, тH. 1943. Genetics of natural populations. IX. Genetics, 28, I62-1 86.

Dobzhansky, Th. 1944. Chromosomal races in Drosophila pseudoobscura and Drosophila persimilis. Carnegie Inst. Washington, Publ. 554, 47-I 44 .

Dobzhansky, тн. 1947. Genetics of natural populations. XIV. Genetics, 32, I 42 - 160 .

Dobzhansky, тн. 1950. Genetics of natural populations. XIX. Genetics, 35, 288-302.

Dobzhansky, тн. 1956. Genetics of natural populations. XXV. Evolution, ro, 82-92.

DOBZHANSKY, TH., AND LEVENE, H. 1948. Genetics of natural populations. XVIII. Genetics, 33, 537-547.

DOBZHANSKY, TH., AND PAVlovsKy, o. 1955. An extreme case of heterosis in a Central American population of Drosophila tropicalis. P.N.A.S., 4I, 289-295.

DOBZHANSKY, TH., AND SPASSKY, N. 1954. Environmental modification of heterosis in Drosophila pseudoobscura. P.N.A.S., 40, 407-415.

EPLiNG, C., MITGhell, D. F., AND MATtONI, R. H. T. I953. On the role of inversions in wild populations of Drosophila pseudoobscura. Evolution, 7, 342-365.

EPLING, C., Mitchell, D. F., AND MATTONI, R. H. T. 1957. The relation of an inversion system to recombination in wild populations. Evolution, $I I, 225-247$.

HALDANE, J. B. s. 1956. The conflict between inbreeding and selection. I. Selffertilization. 7. Genet., 54, 56-63.

HAYMAN, B. I. 1953. Mixed selfing and random mating when homozygotes are at a disadvantage. Heredity, 7, 185-192.

HAYMAN, B. I., AND MATHER, K. 1953. The progress of inbreeding when homozygotes are at a disadvantage. Heredity, $7,165-183$.

HAYMAN, B. I., AND MATHER, K. 1956 . Inbreeding when homozygotes are at a disadvantage, a reply. Heredity, ro, 271-274.

HEUTS, M. J. I947. Influence of humidity on the survival of different chromosomal types in Drosophila pseudoobscura. P.N.A.S., 33, 210-2 13.

HEUTS, M. J. 1948. Adaptive properties of carriers of certain gene arrangements in Drosophila pseudoobscura. Heredity, 2, 63-75.

KIMURA, м. I954. Process leading to quasi-fixation of genes in natural populations due to random fluctuations of selection intensities. Genetics, 39, 280-295. 
KImURA, M. I955. Stochastic processes and distribution of gene frequencies under natural selection. Cold Spring Harbor Symp. Quant. Biol., 20, 33-52.

LEVENE, H., PAVLOVSKY, O., AND DOBZHANSKY, TH. 1954. Interaction of the adaptive values in polymorphic experimental populations of Drosophila pseudoobscura. Evolution, 8, 335-349.

Levitan, м. I951. Experiments on chromosomal variability in Drosophila robusta. Genetics, 36, 285-305.

Levitan, M. 1954. Position effects in natural populations. Amer. Natur., 88, 4 I9-423.

LEVITAN, M. 1955. Studies on linkage in populations. Evolution, 9, 62-74.

MALÉCOT, M. G. 1954. Sur une problème de probabilités en chaîne que pose la génétique. Comptes Rendus A.S., 219, 379-381.

MALÉCOT, M. G. 1955. La diffusion des gènes dans une population mendélicnne. Comptes Rendus A.S., 22I, 340-342.

PaVAn, C., DOBzhansky, Th., AND DA cunha, A. B. 1957. Heterosis and elimination of weak homozygotes in natural populations of three related species of Drosophila. P.N.A.S., 43, 226-234.

REEVE, E. C. R. I955. Inbreeding with the homozygotes at a disadvantage. Am. Human Genetics, 19, 332-346.

Rosenbaum-moos, J. 1955. Comparative physiology of some chromosomal types in Drosophila pseudoobscura. Evolution, 9, $\mathrm{I} 4 \mathrm{I}^{\mathrm{I}-\mathrm{I}} 5^{\mathrm{I}}$.

SPIESS, E. B., KeTChel, M., AND KinNe, B. P. I952. Physiological properties of gene arrangement carriers in Drosophila persimilis. Evolution, 6, 208-2 I 5 .

SPIEss, E. B., AND SChuellein, R. J. I 956 . Chromosomal adaptive polymorphism in Drosophila persimilis. Genetics, $41,50 \mathrm{I}-5 \mathrm{I} 6$.

WRIGHT, S. I93I. Evolution in Mendelian populations. Genetics, IÓ, 97-I59.

WRIGHT, s., AND DOBZHANSKY, TH. 1946. Genetics of natural populations. XII. Genetics, 3I, I26-156. 